\title{
Fermented milk containing Bifidobacterium lactis DN-173 010 improves gastrointestinal well-being and digestive symptoms in women reporting minor digestive symptoms: a randomised, double-blind, parallel, controlled study
}

\author{
Denis Guyonnet ${ }^{1}$, Armelle Schlumberger ${ }^{1}$, Leila Mhamdi $^{1}$, Stefan Jakob ${ }^{1}$ and Olivier Chassany ${ }^{2}$ \\ ${ }^{1}$ Danone Research, Palaiseau, France \\ ${ }^{2}$ Clinical Research, Assistance Publique Hopitaux de Paris \& Denis-Diderot University, Paris, France
}

(Received 1 December 2008 - Revised 21 May 2009 - Accepted 29 May 2009 - First published online 22 July 2009)

\begin{abstract}
The ability of probiotics to improve bowel habits or transit time has been shown in healthy populations. Additional data are required to support the use of specific probiotics to improve gastrointestinal (GI) well-being. The present study was designed to investigate the effect of consuming fermented milk (FM) on GI well-being, digestive symptoms and health-related quality of life (HRQoL) amongst women without diagnosed GI disorders. In this double-blind, controlled, parallel-design study, subjects were randomised to ingest daily either $2 \times 125 \mathrm{~g}$ FM containing Bifidobacterium lactis DN-173 010 and yoghurt strains or a control non-fermented dairy product for 4 weeks followed by a 4-week wash-out period. GI well-being and digestive symptoms were assessed weekly. HRQoL was measured every 4 weeks. Data were analysed using analysis of covariance and logistic regression, correcting for baseline values on the full analysis set population of 197 women (aged 18-60 years). The percentage of women reporting an improvement in their GI well-being was significantly $(P<0.01)$ higher in the FM group $v$. the control group (OR 1.69; $95 \%$ CI 1.17, 2.45). A significantly $(P<0.05)$ more pronounced decrease in the composite score of digestive symptoms was observed in the FM group when comparing with the control group (least squares mean $-0.57 ; 95 \% \mathrm{CI}-1 \cdot 12,-0 \cdot 02$ ). Among HRQoL dimensions, the digestive comfort score was significantly $(P<0.05)$ improved in the FM group compared with the control group. The present study showed that the daily consumption of a specific FM is able to improve GI well-being and digestive symptoms in adult women without GI disorders.
\end{abstract}

Probiotics: Gastrointestinal tract: Adults: Randomised controlled trials

Clinical evidence of the efficacy of some probiotics in the improvement of symptoms in irritable bowel syndrome (IBS) has recently emerged ${ }^{(1-3)}$. The variability of the effects reported has highlighted the product and strain specificity, which is supported by the high variability in their properties (anti-inflammatory activity, effect on motility or visceral sensation $)^{(1,2)}$. However, due to the complexity of the pathophysiology of IBS which remains poorly understood to date, specific research is required to demonstrate these benefits in a more general population with minor digestive complaints. Only a few studies have been focused on the effects of probiotics on gastrointestinal (GI) well-being in a general population. Most of these studies have investigated the effect of probiotics on bowel habits and were conducted in small sample sizes (from thirty to eighty subjects). Improvement of stool frequency and/or volume or weight, and stool consistency has been shown ${ }^{(4-8)}$. Unfortunately, only one study ${ }^{(4)}$ has investigated the impact on a specific digestive symptom (i.e. flatulence), showing a positive effect on this parameter; therefore, the real end-benefits of such functional changes for the consumers are open to question.
Studies in human subjects have been designed to investigate the effect of a fermented milk (FM) product containing Bifidobacterium lactis DN-173 010 and yoghurt starters Lactobacillus bulgaricus and Streptococcus thermophilus. The ability of the strain B. lactis DN-173 010 to survive through the entire digestive tract has been shown in human subjects $^{(9-12)}$, demonstrating the ability of this strain to exert its health effect all along the GI tract. This probiotic food has been shown to improve digestive comfort and symptoms in IBS with predominant constipation (IBSC) ${ }^{(13,14)}$ as well as transit time in both healthy ${ }^{(15-17)}$ and IBS-C populations ${ }^{(13)}$. These data support the ability of this specific FM to improve some GI functions in both healthy and IBS populations.

While the effect of probiotics on GI well-being and digestive symptoms has been widely investigated in IBS, only the impact on bowel function has been studied in healthy populations. The present randomised, double-blind, controlled clinical trial was designed to assess the effects of a FM on GI well-being, digestive symptoms and health-related quality of life (HRQoL) among adult women without GI disorders.

Abbreviations: FAS, full analysis set; FBA, Food and Benefits Assessment; FM, fermented milk; GI, gastrointestinal; HRQoL, health-related quality of life; IBS, irritable bowel syndrome; MID, minimal important difference; PGWBI, Psychological General Well-Being Index.

* Corresponding author: Dr Denis Guyonnet, fax +3316935 76 96, email denis.guyonnet@ danone.com 


\section{Experimental methods}

\section{Study population}

Subjects ( $n$ 240) were recruited in Germany from one clinical centre (Harrison clinical centre, Munich). They were all women, aged 18-60 years, with normal weight or overweight (BMI $18-30 \mathrm{~kg} / \mathrm{m}^{2}$ ), without a diagnosis of any digestive disease including functional bowel disorders such as IBS. Only individuals with a bowel movement frequency within the normal range (3-21 per week) were recruited. At study entry, a screening questionnaire was used to determine the frequency of four different digestive symptoms (i.e. discomfort or abdominal pain, bloating, flatulence/passage of gas, borborygmi/rumbling stomach) in the past month. Frequency of each symptom was assessed using a six-point Likert scale (never $=0 ; 1 \mathrm{~d}$ per month $=1 ; 2-3 \mathrm{~d}$ per month $=2$; $1 \mathrm{~d}$ per week $=3 ;>1 \mathrm{~d}$ per week $=4$; every day $=5$ ) leading to an overall symptom score ranging from 0 (no symptoms) to 20 (all symptoms every day). To be considered for inclusion, all subjects had to have a digestive symptoms score between 8 and 16 or at least one digestive symptom with a score $>4$. Subjects were usual consumers of dairy products.

Subjects were excluded from the study if they had any significant systemic disease or if they were under prescription for medication for digestive symptoms. Antibiotic ingestion within the month before the entry in the study was also an exclusion criterion.

Individuals with known lactose intolerance or with dietary habits which might interfere with the assessment of the study product (for example, slimming or vegetarian diets) or known allergy to the study product components were also excluded.

Throughout the study, the subjects were not allowed to consume any probiotic (including food supplements) or fermented dairy product other than those provided. They were encouraged to continue with all the other aspects of their dietary and physical exercise habits.

\section{Study protocol}

The study was a single-centre, randomised, double blind, controlled, parallel-group study assessing the effect of daily consumption of a FM containing B. lactis DN-173 010 (FM group) $v$. a non-fermented dairy product (control group).

During a 2-week run-in period, baseline values were obtained for the outcome parameters with a weekly assessment of frequency of digestive symptoms and bowel function (bowel movement and stool consistency). The questionnaire used for the assessment of the frequency of digestive symptoms (abdominal pain/discomfort, bloating, flatulence/passage of gas and borborygmi/rumbling stomach) during this run-in period was different to the screening questionnaire used at study entry. The frequency of each individual digestive symptom was evaluated weekly with a five-point Likert scale that ranges from 0 (never) to 4 (every day of the week) leading to a composite score ranging from 0 (no symptoms) to 16 (all symptoms every day). Only subjects having a mean score between 2 and 12 during this run-in period and also meeting the other randomisation criteria (normal bowel movement frequency, no consumption of antibiotics) were randomised to consume during 4 weeks two products per $\mathrm{d}$ (one at breakfast and one at the evening meal) in a doubleblind trial. After this first 4-week period, subjects entered a 4-week period of wash-out without consumption of any specific product (for study flow design, see Fig. 1). Thereafter, subjects started another 4-week period in which all subjects consumed the test product (two servings per d). During this open period, all subjects were informed that they consumed the test product without knowing the commercial name of the product. The purpose of this open period was to specifically investigate the potential placebo effect. Results of this period will be published elsewhere.

The study protocol was conducted in accordance with the Declaration of Helsinki and approved by the Ethics Committee Bayerische Landesaerztekammer (Munich, Germany). All volunteers gave written informed consent before inclusion in the study.

\section{Study products}

The test product was a FM (Activia ${ }^{\circledR}$; Danone) containing B. lactis DN-173 010 (strain number I-2494 in the French National Collection of Cultures of Micro-organisms (CNCM), Paris, France) together with the two classical yoghurt starters, S. thermophilus (CNCM strain number I-1630) and L. bulgaricus (CNCM strain numbers I-1632 and I-1519), and Lactococcus cremoris (CNCM strain number I-1631). The test product contains $1.25 \times 10^{10}$ colony-forming units (cfu) B. lactis DN-173 010 per serving and $1.2 \times 10^{9}$ cfu $S$. thermophilus and L. bulgaricus per serving.

The control product was a milk-based non-fermented dairy product without probiotics and with a low content of lactose ( $<4 \mathrm{~g} /$ serving), which is similar to the content of lactose in the test product. The control product was acidified using an enzymic process which mimics the acidification process that occurs during the fermentation of milk by B. lactis and yoghurt symbiosis. Both the test and control products were without flavour and had a similar appearance, texture and taste. Each serving contained $125 \mathrm{~g}$. Both products were specifically prepared for the study and provided by Danone Research (Palaiseau, France).

Compliance was calculated on the basis of the data reported by subjects in their diaries and the number of non-used servings returned.

\section{Assessments and study endpoints}

Gastointestinal well-being. The overall assessment of GI well-being was self-evaluated by subjects weekly from the first week of product consumption (week 1) to the end of the study with the following question: 'How do you consider in the past seven days, your GI well-being (intestinal transit, stool frequency and consistency, abdominal pain/discomfort, bloating, flatulence/passage of gas, borborygmi/rumbling stomach) compared to the period before beginning the consumption of the study product?'. This global rating is a combined scale leading to a fifteen-point Likert scale $(-7,0,+7)$. In a first step, subjects indicate if their GI well-being has remained the same, improved, or worsened (three-point Likert scale) and in a second step, subjects with 


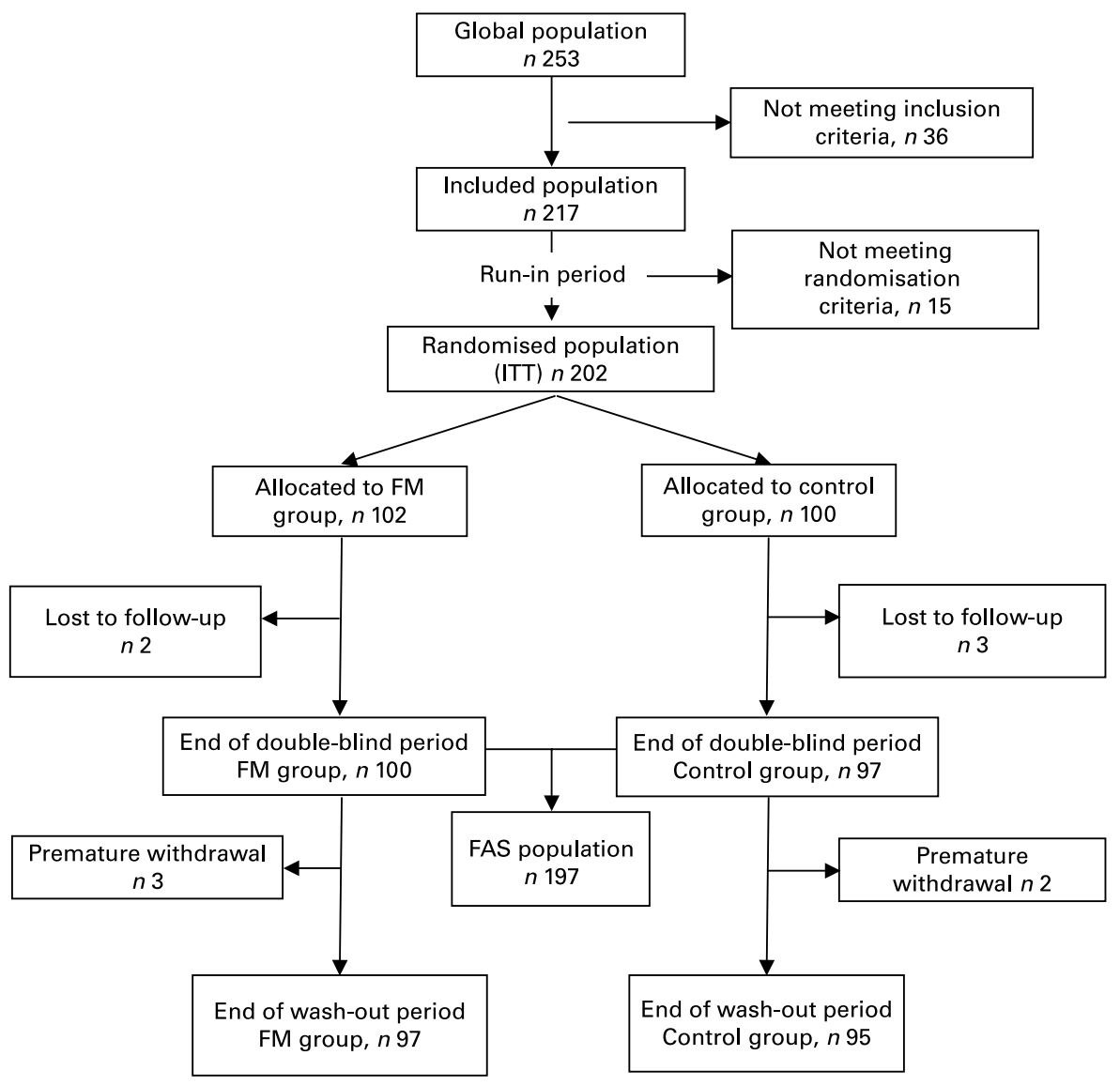

Fig. 1. Study flow design. ITT, intention-to-treat population (defined as all randomised subjects); FM, fermented milk; FAS, full analysis set population (defined as all randomised subjects having any efficacy data available under product consumption).

improving or worsening symptoms rate the degree of change on a seven-point scale. This scale was adapted from the scale developed by Guyatt et al. ${ }^{(18)}$ and used in IBS studies $^{(19)}$.

As recommended in guidelines for the design of treatment trials for functional GI disorders ${ }^{(19)}$, each subject was classified as a responder or a non-responder. The definition of a responder was based on the recommendations for global assessment of symptom relief in IBS trials ${ }^{(19)}$, which is the only available recommendation for such kinds of assessment. A responder was defined as a subject having an improvement in their GI well-being, i.e. answering 'improved' on the threepoint Likert scale, on at least 2 weeks over the 4-week double-blind period of product consumption. The same criterion was applied to define the responders for the 4-week wash-out.

Digestive symptoms. The frequency of four individual digestive symptoms (abdominal pain/discomfort, bloating, flatulence/passage of gas and borborygmi/rumbling stomach) was evaluated weekly with a five-point Likert scale that ranges from 0 (never) to 4 (every day of the week) throughout the study. A composite score was calculated ranging from 0 to 16 .

Bowel function. Bowel movements were reported daily throughout the study as well as stool consistency for each stool passed according to the Bristol stool form scale ${ }^{(20)}$. In order to assess the normalisation of stool consistency, scores of stool consistency were recoded as follow: $0=$ type 4 (like a sausage or snake, smooth and soft); $1=$ types 3 (like a sausage but with cracks on surface) and 5 (soft blobs with clear-cut edges); $2=$ types 2 (sausage shaped but lumpy) and 6 (fluffy pieces with ragged edges, a mushy stool); 3 = types 1 (separate hard lumps like nuts, difficult to pass) and 7 (watery, no solid pieces, entirely liquid).

Health-related quality of life. HRQoL of subjects was assessed by self-administration of two questionnaires, the Food and Benefits Assessment (FBA) ${ }^{(21)}$ and the Psychological General Well-Being Index (PGWBI) ${ }^{(22)}$. The questionnaires were completed at baseline and after 4 and 8 weeks corresponding to the end of the periods of product consumption and of the wash-out period, respectively.

The FBA questionnaire has been developed and validated according to international recognised guidelines used for patient-reported outcomes and aims at assessing specifically the benefits of a food or a diet on HRQoL. This questionnaire comprises forty-one items, making it possible to calculate scores for seven dimensions (snacking, vitality, well-being, physical appearance, aesthetics, digestive comfort and disease prevention). The scores range from 0 to 100 (best).

The PGWBI (a generic questionnaire) measures psychological well-being and distress and is composed of twenty-two items which constitute six dimensions (anxiety, depression, self-control, positive well-being, general health and vitality). The scores of all dimensions can be summarised to provide a global score ${ }^{(22)}$. The scores range from 0 to 100 (best). 
The FBA digestive comfort dimension score and the PGWBI global score were defined as the main scores for HRQoL analysis. Other dimensions of both questionnaires were considered as secondary HRQoL criteria.

The magnitude of the changes in the digestive comfort dimension of the FBA was assessed in two ways: (i) clinical relevance of the difference in the score changes between groups; (ii) comparison of rate of responders. This was done using the minimal important difference (MID) method, which is one of the methods allowing the magnitude of the effects in HRQoL ${ }^{(23)}$ to be ascertained ${ }^{(24)}$. The MID corresponds to the minimal difference in the digestive comfort score for which the subjects perceived a benefit. The populations used to calculate the MID values were: (i) subjects reporting an improvement of their GI well-being at week 4 of +2 or +3 ; (ii) subjects reporting an improvement of their GI well-being at week 4 of +4 or +5 . Finally, the difference in the score changes between groups was considered as clinically significant if this difference was superior to the MID value (i) and a responder will be defined as a subject having an improvement in their baseline score of digestive comfort dimension of at least the value of the MID (ii).

Subjects recorded daily in their diary the consumption of study products, medications started during the study and forbidden products (fibres, other fermented dairy products), as well as any adverse events.

\section{Statistical methods}

The sample-size calculation was based on the main outcome, the overall assessment of GI well-being over the first 4 weeks of the study (evaluated with a three-point Likert scale) for the main criteria (ordered categorical data). The trial sample size required to give a power of $80 \%$ for detecting a significant difference between the control and test products of $20 \%$ more subjects improved in the test-product group at 4 weeks was calculated to be at least eighty-four subjects per group. Taking into account that all subjects who withdrew prematurely were not replaced, 100 subjects per product group were randomised.

The intention-to-treat population corresponds to all the randomised subjects. All the analyses of efficacy were performed on the full analysis set (FAS) population which corresponds to all randomised subjects having any efficacy data available under product consumption. This definition of the FAS population is in agreement with the International Conference of Harmonisation guidances ${ }^{(25)}$.

Baseline demographic data were compared between groups using the Wilcoxon test. Overall assessment of GI well-being (fifteen-point scale), frequency of digestive symptoms, and stool frequency and consistency were analysed using a repeated-measures ANOVA (on raw data or on change from baseline or on the ranks according to the normality assessment) with time, treatment group, interaction time $\times$ product and baseline score as fixed factors for each period.

One-factor non-parametric ANOVA based on the ranks was carried out by time, i.e. by week, when the normality of the residual of the repeated-measures parametric or non-parametric model analysis over the 4-week period was not assessed.
The measures on the three-point scale of overall assessment of GI well-being are multinomial and repeated, so in this case the methodology used is the generalised estimation equations analysed with the GENMOD procedure.

The OR was used to report GI well-being results and corresponds to the odds to be in a better condition for the overall assessment of GI well-being and to the odds to be responder for GI well-being in the FM group $v$. the control group.

The analysis of the score differences for all the dimensions of the FBA and PGWBI questionnaires, at weeks 4 and $8 v$. baseline, between the FM and control groups was done using a parametric or non-parametric covariance analysis according to the normality of the assessment and the model residuals (treatment group and baseline score as fixed factors and primary care centre as a random factor).

The responder rates for overall assessment of GI well-being, the digestive comfort dimension of the FBA, were analysed at weeks 4 and 8 by a logistic regression to compare the products.

Analyses during the wash-out period were performed only on parameters for which significant differences were shown during the double-blind period.

\section{Results}

\section{Subjects}

Figure 1 describes the flow of subjects through the protocol. From the 253 contacted subjects, 217 were included in the present study and 202 were randomised (102 subjects assigned to the FM group and 100 to the control group), corresponding to the intention-to-treat population. Of the subjects, five did not complete the entire 4-week double-blind period and were lost to follow-up (no data available under product consumption), giving an FAS population of 197. Of these, five additional subjects prematurely stopped the study during the wash-out period.

The compliance during the study was 99.2 and $99.3 \%$ for the control and FM groups, respectively.

\section{Baseline characteristics of subjects}

The subjects showed no significant differences between the FM product and control groups (Table 1).

\section{Double-blind period}

Gastrointestinal well-being. The main outcome (i.e. overall assessment of GI well-being over the 4-week period) was analysed on the three-point scale level of this assessment (Table 2). The percentage of women reporting an improvement in their GI well-being was significantly $(P=0.006)$ higher in the FM group $v$. the control group (OR 1.7; $95 \%$ CI $1 \cdot 17,2.45)$. The weekly analysis of the fifteen-point scale score showed a significant higher score at weeks 1 $(P=0 \cdot 001)$ and $3(P=0 \cdot 047)$ in the FM group compared with the control group.

The percentage of responders for GI well-being was significantly $(P=0.025)$ higher in the FM group $v$. the control group $(52.0$ v. $36 \cdot 1 \%$, respectively; OR $1.92 ; 95 \%$ CI $1.09,3.40)$ (Table 2). 
Table 1. Baseline characteristics of subjects: comparison between groups

(Mean values and standard deviations)

\begin{tabular}{|c|c|c|c|c|c|}
\hline & \multicolumn{2}{|c|}{$\begin{array}{l}\text { FM group } \\
(n \text { 100) }\end{array}$} & \multicolumn{2}{|c|}{$\begin{array}{c}\text { Control group } \\
(n 97)\end{array}$} & \multirow[b]{2}{*}{$P^{*}$} \\
\hline & Mean & SD & Mean & SD & \\
\hline Age (years) & 31.9 & $9 \cdot 2$ & 32.5 & $10 \cdot 5$ & NS \\
\hline Age range (years) & \multicolumn{2}{|c|}{$18-57$} & \multicolumn{2}{|c|}{$18-59$} & \\
\hline BMI $\left(\mathrm{kg} / \mathrm{m}^{2}\right)$ & $23 \cdot 3$ & $2 \cdot 7$ & $23 \cdot 2$ & 2.5 & NS \\
\hline Borborygmi & 1.60 & 0.90 & 1.57 & 0.91 & NS \\
\hline Bloating† & 1.95 & 0.80 & $2 \cdot 15$ & 0.87 & NS \\
\hline Flatulence $†$ & 2.48 & 0.81 & 2.48 & 0.83 & NS \\
\hline Abdominal pain $†$ & 1.00 & 0.77 & 1.05 & 0.73 & NS \\
\hline Composite score $\ddagger$ & 7.03 & 2.09 & $7 \cdot 25$ & $2 \cdot 27$ & NS \\
\hline $\begin{array}{l}\text { FBA digestive comfort } \\
\text { score§ }\end{array}$ & $65 \cdot 3$ & $13 \cdot 6$ & 65.5 & $12 \cdot 2$ & NS \\
\hline Stool frequency & $7 \cdot 32$ & $3 \cdot 12$ & $7 \cdot 25$ & 2.92 & NS \\
\hline Stool consistency\| & 1.30 & 0.52 & 1.29 & 0.58 & NS \\
\hline
\end{tabular}

FM, fermented milk; FBA, Food and Benefits Assessment.

* No significant differences were found between the groups for all the variables tested.

†The frequency of individual digestive symptoms was assessed with a five-point Likert scale from 0 (never) to 4 (every day of the week).

$¥$ The composite score of the frequency of digestive symptoms ranged from 0 to 16 . $\S$ The FBA questionnaire digestive comfort score ranged from 0 to 100 (best).

॥ Stool consistency ranged from 0 to 3 .

Frequency of digestive symptoms. Weekly changes during the period of product consumption, the mean change over this 4-week period for the frequency of each individual digestive symptoms, as well as composite scores within the FM and double-blind and the wash-out periods control groups are shown in Table 3 . Changes in borborygmi frequency showed a significantly $(P=0 \cdot 016)$ higher decrease in the FM group compared with the control group over the 4-week period (least squares mean -0.22 ; $95 \%$ CI -0.40 , $-0 \cdot 04)$. The decrease in flatulence frequency was significantly higher in the FM group than in the control group at weeks 1 $(P=0.041), 2(P=0.028)$ and $4(P=0.008)$, whereas no significant differences were observed in bloating score as well as in abdominal pain or discomfort score. Comparisons of the effect of the test product on a composite score showed an overall significant $(P=0 \cdot 044)$ decrease over the 4-week period in the FM group when comparing with the control group (least squares mean $-0.57 ; 95 \%$ CI $-1 \cdot 12,-0.02)$.

\section{Bowel function}

Stool frequency and stool consistency data are shown in Table 4. Stool frequency did not differ between the FM and control groups. A significant $(P=0.02)$ decrease of the stool consistency was observed in the FM group $v$. the control group.

Health-related quality of life. Results of the FBA digestive comfort dimension score, and of the PGWBI global score are shown in Table 5. The digestive comfort dimension score, the primary HRQoL endpoint, significantly increased $(P=0.027)$ after 4 weeks of product consumption in the FM group as compared with the control group. The observed difference between group (3.56) is inferior to the MID $(10 \cdot 9)$. When the rate of responders for the digestive comfort

Table 2. Overall assessment of gastrointestinal (GI) well-being in the full analysis set population ( $n$ 197) during the

\begin{tabular}{|c|c|c|c|c|c|c|c|c|}
\hline & \multicolumn{4}{|c|}{ Double-blind period } & \multicolumn{4}{|c|}{ Wash-out period } \\
\hline & Week 1 & Week 2 & Week 3 & Week 4 & Week 5 & Week 6 & Week 7 & Week 8 \\
\hline \multicolumn{9}{|l|}{ Percentage by class $\dagger$} \\
\hline Worsened & 7 & 7 & 5 & 6 & 20 & 30 & 26 & 19 \\
\hline No change & 56 & 56 & 54 & 53 & 68 & 59 & 64 & 70 \\
\hline Improved & 37 & 37 & 41 & 41 & 11 & 10 & 8 & 6 \\
\hline \multicolumn{9}{|l|}{ Control group ( $n$ 97) } \\
\hline Worsened & 19 & 19 & 12 & 17 & 13 & 22 & 20 & 11 \\
\hline No change & 60 & 60 & 54 & 50 & 63 & 61 & 65 & 75 \\
\hline Improved & 22 & 22 & 34 & 34 & 20 & 13 & 11 & 8 \\
\hline OR & \multirow{2}{*}{\multicolumn{4}{|c|}{$\begin{array}{c}1 \cdot 69^{\star *} \\
1.17,2 \cdot 45\end{array}$}} & \multirow{2}{*}{\multicolumn{4}{|c|}{ NS }} \\
\hline $95 \% \mathrm{Cl}$ & & & & & & & & \\
\hline \multicolumn{9}{|l|}{ Raw scoresł } \\
\hline \multicolumn{9}{|l|}{ FM group $(n 100)$} \\
\hline Mean & $1 \cdot 21^{\star \star}$ & 1.36 & $1.41^{*}$ & 1.37 & -0.37 & -0.57 & -0.65 & -0.42 \\
\hline SD & $2 \cdot 13$ & $2 \cdot 21$ & $2 \cdot 40$ & 2.54 & $2 \cdot 08$ & $2 \cdot 17$ & $2 \cdot 00$ & 1.59 \\
\hline \multicolumn{9}{|l|}{ Control group ( $n$ 97) } \\
\hline Mean & $0 \cdot 10$ & 0.75 & 0.60 & 0.80 & 0.30 & -0.13 & -0.28 & 0.00 \\
\hline SD & $2 \cdot 16$ & $2 \cdot 21$ & $2 \cdot 49$ & $2 \cdot 33$ & 2.07 & 2.01 & 2.06 & 1.42 \\
\hline \multicolumn{9}{|l|}{ Rate of responders $(\%) \S$} \\
\hline FM group $(n 100)$ & \multicolumn{4}{|c|}{$52 \cdot 0$} & \multicolumn{4}{|c|}{8.0} \\
\hline Control group ( $n$ 97) & \multicolumn{4}{|c|}{$36 \cdot 1$} & \multicolumn{4}{|c|}{$11 \cdot 3$} \\
\hline OR & \multirow{2}{*}{\multicolumn{4}{|c|}{$\begin{array}{c}1.92^{*} \\
1.09,3 \cdot 40\end{array}$}} & \multirow{2}{*}{\multicolumn{4}{|c|}{ NS }} \\
\hline $95 \% \mathrm{Cl}$ & & & & & & & & \\
\hline
\end{tabular}

FM, fermented milk; NS, OR not statistically different $(P>0.05)$, with $\mathrm{OR}<1$.

${ }^{*} P<0.05,{ }^{* *} P<0.01$.

$\dagger$ Analysis of the percentage of subjects in a higher class was done using the GENMOD procedure.

‡ Raw scores ranged from -7 to +7 . Analysis of the raw scores was done by week using rank analysis for each time.

$\S A$ responder was defined as a subject having an improvement of their Gl well-being for at least 2 weeks among the 4 weeks. Analysis of the rate of responders was done using a logistic model. 
Table 3. Changes in frequency of digestive symptoms in the full analysis set population ( $n$ 197) during the 4 weeks of the double-blind period $\dagger$ (Mean values and standard deviations)

\begin{tabular}{|c|c|c|c|c|c|c|c|c|c|c|}
\hline & \multicolumn{8}{|c|}{ Week } & \multirow[b]{3}{*}{ Least squares mean } & \multirow[b]{3}{*}{$95 \% \mathrm{Cl}$} \\
\hline & \multicolumn{2}{|c|}{1} & \multicolumn{2}{|c|}{2} & \multicolumn{2}{|c|}{3} & \multicolumn{2}{|c|}{4} & & \\
\hline & Mean & SD & Mean & SD & Mean & SD & Mean & SD & & \\
\hline Borborygmił & & & & & & & & & $-0.22^{*}$ & $-0.40,-0.04$ \\
\hline FM group & -0.25 & 0.91 & -0.45 & 0.98 & -0.62 & 0.90 & -0.70 & 0.95 & & \\
\hline Control group & -0.09 & 0.87 & -0.28 & 0.98 & -0.31 & 0.95 & -0.33 & 0.95 & & \\
\hline Bloating $\ddagger$ & & & & & & & & & -0.13 & $-0.32,0.06$ \\
\hline FM group & -0.36 & 0.87 & -0.59 & 0.91 & -0.64 & 0.98 & -0.69 & 0.94 & & \\
\hline Control group & -0.34 & 0.93 & -0.47 & 0.93 & -0.65 & 0.95 & -0.70 & 0.94 & & \\
\hline Abdominal pain $\ddagger$ & & & & & & & & & NV & \\
\hline FM group & -0.14 & 0.77 & -0.43 & 0.84 & -0.39 & 0.92 & -0.37 & 0.88 & & \\
\hline Control group & -0.24 & 0.67 & -0.24 & 0.83 & -0.34 & 0.76 & -0.44 & 0.87 & & \\
\hline Flatulence $\ddagger$ & & & & & & & & & NV & \\
\hline FM group & -0.29 & $0.80^{\star}$ & -0.52 & $0.92^{*}$ & -0.55 & 0.99 & -0.74 & $1.02^{\star}$ & & \\
\hline Control group & -0.06 & 0.86 & -0.23 & 1.03 & -0.29 & 0.92 & -0.42 & 0.96 & & \\
\hline Composite score§ & & & & & & & & & $-0.57^{\star}$ & $-1.12,-0.02$ \\
\hline FM group & -1.06 & $2 \cdot 23$ & -1.99 & 2.57 & $-2 \cdot 20$ & 2.56 & $-2 \cdot 51$ & $2 \cdot 75$ & & \\
\hline Control group & -0.77 & 2.59 & $-1 \cdot 22$ & $2 \cdot 85$ & -1.59 & $2 \cdot 68$ & -1.89 & $2 \cdot 76$ & & \\
\hline
\end{tabular}

FM, fermented milk; NV, not valid.

* $P<0.05$.

†FM group, $n$ 100; control group, $n$ 97. Changes from baselines in the frequency of digestive symptoms and composite score were compared between groups over the 4 weeks (least squares mean) with the mixed linear model. When the statistical model was not valid for the overall comparison (NV), changes from baselines were analysed by week using a non-parametric model.

¥The frequency of individual digestive symptoms was assessed with a five-point Likert scale from 0 (never) to 4 (every day of the week).

$\S$ The composite score of frequency of digestive symptoms ranged from 0 to 16 .

dimension was analysed, a trend in favour of a higher percentage of responders was shown at week 4 in the FM group as compared with the control group (41.0 v. 27.8\%; $P=0.053)$. No difference was observed between groups for other HRQoL dimensions. PGWBI scores did not differ between groups over time.

\section{Wash-out period}

The percentage of women reporting an improvement in their GI well-being was not significantly different in the FM group $v$. the control group as well as for the difference in the weekly analysis of the raw score (Table 2). At the end of the wash-out period, the percentage of responders strongly decreased without difference between the groups $(8.0 \mathrm{v}$. $11.3 \%$ for the FM and control groups, respectively; OR $0.68 ; 95 \%$ CI $0.26,1.77$ ).

No significant difference in the changes of frequency for each individual symptom was observed between groups. Comparisons of the composite score showed an overall significant $(P=0.044)$ decrease over the 4 -week period in the control group when comparing with the FM group.

Stool frequency as well as score of stool consistency did not differ between the FM product and control groups (data not shown). No difference was observed between the groups for all HRQoL dimensions at the end of the wash-out period compared with baseline values (data not shown).

Table 4. Changes in bowel frequency and stool consistency in the full analysis set population $(n 197)$ during the 4 weeks of the double-blind period*

(Mean values and standard deviations)

\begin{tabular}{|c|c|c|c|c|c|c|c|c|c|}
\hline & \multicolumn{8}{|c|}{ Week } & \multirow[b]{3}{*}{$P$} \\
\hline & \multicolumn{2}{|c|}{1} & \multicolumn{2}{|c|}{2} & \multicolumn{2}{|c|}{3} & \multicolumn{2}{|c|}{4} & \\
\hline & Mean & SD & Mean & SD & Mean & SD & Mean & SD & \\
\hline \multicolumn{10}{|l|}{ Bowel frequency } \\
\hline FM group & 1.04 & $2 \cdot 36$ & 0.91 & $2 \cdot 19$ & $1 \cdot 16$ & $2 \cdot 42$ & 1.40 & 3.55 & NS \\
\hline Control group & 0.93 & 2.59 & 1.02 & $2 \cdot 78$ & 0.77 & 2.53 & 0.93 & $2 \cdot 46$ & \\
\hline \multicolumn{10}{|l|}{ Stool consistency† } \\
\hline FM group & -0.13 & 0.53 & -0.23 & 0.55 & -0.32 & 0.58 & -0.35 & 0.55 & $<0.05$ \\
\hline Control group & -0.08 & 0.53 & -0.11 & 0.52 & -0.22 & 0.52 & -0.15 & 0.62 & \\
\hline
\end{tabular}

FM, fermented milk

${ }^{*}$ FM group, $n$ 100; control group, $n$ 97. Changes from baselines were compared between groups over the 4 weeks with mixed linear models on the ranks.

† Stool consistency ranged from 0 to 3 . 
Table 5. Health-related quality of life: digestive comfort dimension score of the Food and Benefits Assessment (FBA) questionnaire and the global score of the Psychological General Well-Being Index (PGWBI) questionnaire in the full analysis set population ( $n$ 197) at baseline and end of the double-blind period

(Mean values and standard deviations)

\begin{tabular}{|c|c|c|c|c|c|c|c|c|c|c|c|c|c|c|}
\hline & \multicolumn{6}{|c|}{ FM group ( $n 100)$} & \multicolumn{6}{|c|}{ Control group ( $n$ 97) } & \multirow{2}{*}{\multicolumn{2}{|c|}{$\begin{array}{c}\text { Difference in } \\
\text { change } \\
\text { between } \\
\text { groups } †\end{array}$}} \\
\hline & \multicolumn{2}{|c|}{ Baseline } & \multicolumn{2}{|c|}{ Week 4} & \multicolumn{2}{|c|}{ Change } & \multicolumn{2}{|c|}{ Baseline } & \multicolumn{2}{|c|}{ Week 4} & \multicolumn{2}{|c|}{ Change } & & \\
\hline & Mean & SD & Mean & SD & Mean & SD & Mean & SD & Mean & SD & Mean & SD & Mean & SD \\
\hline FBA digestive comfort‡ & $\begin{array}{l}65 \cdot 3 \\
73.2\end{array}$ & $13 \cdot 6$ & $\begin{array}{l}76 \cdot 0 \\
77 \cdot 2\end{array}$ & $12 \cdot 0$ & $\begin{array}{r}10 \cdot 7 \\
4.0\end{array}$ & $\begin{array}{r}14.2 \\
9.2\end{array}$ & $\begin{array}{l}65 \cdot 5 \\
73.3\end{array}$ & $12 \cdot 2$ & $72 \cdot 6$ & $12 \cdot 2$ & $7 \cdot 1$ & $\begin{array}{c}12 \cdot 2 \\
9.62\end{array}$ & $3.56^{*}$ & 1.59 \\
\hline PGWBI global score§ & $73 \cdot 2$ & $11 \cdot 6$ & $77 \cdot 2$ & $12 \cdot 2$ & $4 \cdot 0$ & $9 \cdot 2$ & $73 \cdot 3$ & 11.9 & $77 \cdot 7$ & $10 \cdot 8$ & 4.4 & 9.62 & NS & \\
\hline
\end{tabular}

FM, fermented milk.

${ }^{*} P<0.05$.

† Changes from baselines in the scores were compared between groups with the mixed linear model.

$\ddagger$ The digestive comfort score ranged from 0 to 100 (best).

$\S$ The global score ranged from 0 to 100 (best).

\section{Discussion}

The present randomised, double-blind, controlled clinical study shows the ability of the tested FM to improve GI well-being in women reporting minor digestive disorders. This beneficial effect is supported by both the OR (1.7) for the improvement of GI well-being and the $15 \%$ difference in the rate of responders for GI well-being over the control. Furthermore, this probiotic product decreased the composite score of the frequency of GI symptoms. This overall symptom improvement was mainly due to improvements in several individual gas-related symptoms (for example, borborygmi and flatulence).

When studying the literature, it can be concluded that it is indeed difficult to perform clinical studies assessing the impact of food and probiotics on GI well-being in the general population. Several studies have reported effects of probiotics on bowel function in healthy populations ${ }^{(4-7,15)}$ but none of them has assessed the effect on digestive comfort or GI symptoms. Indeed, research has focused on the assessment of potential negative sensations from the gut associated with the consumption of both fibres ${ }^{(26)}$ and probiotics ${ }^{(27)}$. Subjectreported assessment is endorsed to be the more meaningful and accurate measure for GI symptoms as compared with physician-reported assessments ${ }^{(19)}$. Thus, in the present study, an overall assessment of GI well-being was chosen to capture the improvement in different GI symptoms as well as in bowel function, which is in line with recent recommendations for the assessment of probiotics in IBS ${ }^{(28)}$. Assessment of the frequency of digestive symptoms, HRQoL and bowel function were considered as secondary endpoints.

Gut-derived symptoms are part of the normal physiological digestive process and are experienced by the general population ${ }^{(29,30)}$. However, the efficacy of probiotics on digestive symptoms was only reported in the IBS population ${ }^{(31)}$. The present study shows that a specific probiotic food that has been shown to improve digestive symptoms in $\operatorname{IBS}^{(13,14)}$ is also able to improve GI well-being and digestive symptoms in individuals who do not suffer from GI diseases. The comparison of the clinical benefit between these studies can be done using the number needed to treat $(\mathrm{NNT})^{(32,33)}$, which is based on the difference of responders between groups. The NNT of the present study (6.25) is similar to the one observed (5.7) in a previous IBS study ${ }^{(14)}$ and within the same range as NNT calculated (4 and 7.3) in recent reviews of IBS probiotics trials ${ }^{(28,31)}$.

Previous studies investigating the effect of probiotics on the GI tract have mainly shown an increase in bowel frequency ${ }^{(5-8)}$. In the present study, GI well-being and symptoms improved without change of bowel frequency. The fermented dairy product used in the present study is also able to decrease transit time in both healthy ${ }^{(15-17)}$ and IBS populations ${ }^{(13)}$. Several other clinical trials have investigated the effect of either probiotic strain(s) alone, i.e. Propionibacterium ${ }^{(34)}$ and VSL\#3 ${ }^{(35)}$ or $\mathrm{FM}^{(36,37)}$ and have failed to demonstrate a positive effect on transit time. This suggests that the benefits exerted by one probiotic product could not be extrapolated to other probiotics.

HRQoL measures were used to ascertain the relevance of the observed improvement of both GI well-being and symptoms. We acknowledge that despite the significant and evident effect on GI well-being and symptoms, the improvement of HRQoL remains less pronounced and is not so clear-cut. In order to provide meaningful interpretation of the HRQoL changes, the clinical significance of the changes in the digestive comfort dimension of the FBA questionnaire was assessed using a priori definitions of $\mathrm{MID}^{(24)}$. Using this very rigorous method of assessment, a trend in favour of the tested product was obtained for the rate of responders whereas the statistically significant difference in the score changes was below the threshold of clinical relevance. Obviously, it is more difficult to improve HRQL in a non-disease population than in a population suffering from a chronic disease such as a digestive pathology ${ }^{(14)}$. A longer trial is necessary to achieve higher improvement in HRQoL, as this parameter is known to change slowly, especially in this population which presents high baseline scores, i.e. good HRQoL.

Another limitation in the interpretation of the results of the present study is the multiple statistical comparisons. Due to the lack of data in this population, the statistical comparisons between groups for all the outcomes are required to detect a positive effect. Although these data of this explorative study show consistent positive effects across the different outcomes, 
further studies aiming at investigating more specifically some outcomes (for example, GI well-being or HRQoL) are required to confirm these beneficial effect.

The effect of the study product was not maintained after the cessation of its consumption. It was noticeable that a dramatic loss of the effect of the product was observed within the first week of ceasing to use the product whereas a slow but gradual loss of the effect was observed during the following 3 weeks of the follow-up period. Interestingly, it was shown that the level of B. lactis in the faeces of healthy volunteers consuming the study product followed a similar kinetics, with a maximal level reached after 4 weeks of consumption followed by a decrease within a week after discontinuation of use of the product and a non-detectable level after $4-5$ weeks $^{(38)}$. Therefore, the disappearance within the GI tract of the specific B. lactis strain of the study product may support the observed decrease of the effect of this product. Given the fact that alterations of gut microbiota have been identified in $\operatorname{IBS}^{(1,39)}$, investigations aiming at assessing the impact of this product on gut microbiota in healthy population as well as in IBS would allow us to determine whether the effects on GI functions and sensations could be associated with gut microbiota modifications.

In conclusion, the present study demonstrated that this probiotic food containing the specific B. lactis DN-173 010 strain is able to improve GI well-being as well as GI symptoms in a population of women reporting minor digestive disorders. These data, taken together with previous data obtained on GI transit and in IBS, suggest that this specific probiotic food may represent a promising nutritional and safe solution for the management of GI symptoms. Future studies are required to explore longer durations of product consumption and the impact on HRQoL. Investigations of gut microbiota modifications will allow the determination of mechanisms of action and could allow the determination of which population could benefit from the consumption of such kinds of probiotics.

\section{Acknowledgements}

The authors thank Stéphane Doat for providing products and Dr De La Motte and Dr Hausleiter for the management of the experimental phase.

The present study was supported by a grant from Danone.

D. G. was responsible for the study design and for the analysis of the study results. S. J. and O. C. were involved in the study design definition and in the analysis of the results. L. M. was responsible for the statistical analysis. A. S. was responsible for the overall study management.

D. G., A. S. and S. J. are employees of Danone Group. O. C. is a consultant of Danone Research.

\section{References}

1. Quigley EM \& Flourie B (2007) Probiotics and irritable bowel syndrome: a rationale for their use and an assessment of the evidence to date. Neurogastroenterol Motil 19, 166-172.

2. Spiller P (2008) Review Article: probiotics and prebiotics in irritable bowel syndrome (IBS). Aliment Pharmacol Ther 28, 385-396.
3. Camilleri M (2006) Probiotics and irritable bowel syndrome: rationale, putative mechanisms, and evidence of clinical efficacy. J Clin Gastroenterol 40, 264-269.

4. Johansson ML, Nobaek S, Berggren A, et al. (1998) Survival of Lactobacillus plantarum DSM $9843(299 \mathrm{v})$, and effect on the short-chain fatty acid content of faeces after ingestion of a rose-hip drink with fermented oats. Int J Food Microbiol 42, $29-38$.

5. Larsen CN, Nielsen S, Kaestel P, et al. (2006) Dose-response study of probiotic bacteria Bifidobacterium animalis subsp. lactis BB-12 and Lactobacillus paracasei subsp. paracasei CRL-341 in healthy young adults. Eur J Clin Nutr 60, 1284-1293.

6. Olivares M, Diaz-Ropero MA, Gomez N, et al. (2006) Oral administration of two probiotic strains, Lactobacillus gasseri CECT5714 and Lactobacillus coryniformis CECT5711, enhances the intestinal function of healthy adults. Int J Food Microbiol 107, 104-111.

7. Yamano T, Iino H, Takada M, et al. (2006) Improvement of the human intestinal flora by ingestion of the probiotic strain Lactobacillus johnsonii La1. Br J Nutr 95, 303-312.

8. Matsumoto K, Takada T, Shimizu K, et al. (2006) The effects of a probiotic milk product containing Lactobacillus casei strain Shirota on the defecation frequency and the intestinal microflora of sub-optimal health state volunteers: a randomized placebo-controlled cross-over study. Biosci Microflora 25, $39-48$.

9. Duez H, Pelletier C, Cools S, et al. (2000) A colony immunoblotting method for quantitative detection of a Bifidobacterium animalis probiotic strain in human faeces. $J$ Appl Microbiol 88, 1019-1027.

10. Pochart P, Marteau P, Bouhnik Y, et al. (1992) Survival of bifidobacteria ingested via fermented milk during their passage through the human small intestine: an in vivo study using intestinal perfusion. Am J Clin Nutr 55, 78-80.

11. Berrada N, Lemeland JF, Laroche G, et al. (1991) Bifidobacterium from fermented milks: survival during gastric transit. J Dairy Sci 74, 409-413.

12. Rochet V, Rigottier-Gois L, Ledaire A, et al. (2008) Survival of Bifidobacterium animalis DN-173 010 in the faecal microbiota after administration in lyophilised form or in fermented product - a randomised study in healthy adults. $J \mathrm{Mol}$ Microbiol Biotechnol 14, 128-136.

13. Agrawal A, Houghton LA, Morris J, et al. (2009) Clinical trial: the effects of a fermented milk product containing Bifidobacterium lactis DN-173-010 on abdominal distension and gastrointestinal transit in irritable bowel syndrome with constipation. Aliment Pharmacol Ther 29, 104-114.

14. Guyonnet D, Chassany O, Ducrotté P, et al. (2007) Effect of a fermented milk containing Bifidobacterium animalis DN-173 010 on the health-related quality of life and symptoms in irritable bowel syndrome in adults in primary care: a multicentre, randomized, double-blind, controlled trial. Aliment Pharmacol Ther 26, 475-486.

15. Marteau P, Cuillerier E, Méance S, et al. (2002) Bifidobacterium animalis strain DN-173 010 shortens the colonic transit time in healthy women: a double-blind, randomized, controlled study. Aliment Pharmacol Ther 16, 587-593.

16. Méance S, Cayuela C, Raimondi A, et al. (2003) Recent advances in the use of functional foods: effects of the commercial fermented milk with Bifidobacterium animalis strain DN-173 010 and yoghurt strains on gut transit time in the elderly. Microb Ecol Health Dis 15, 15-22.

17. Méance S, Cayuela C, Turchet P, et al. (2001) A fermented milk with a Bifidobacterium probiotic strain DN-173 010 shortened oro-faecal gut transit time in elderly. Microb Ecol Health Dis 13, 217-222. 
18. Guyatt GH, Deyo RA, Charlson M, et al. (1989) Responsiveness and validity in health status measurement: a clarification. $J$ Clin Epidemiol 42, 403-408.

19. Irvine EJ, Whitehead WE, Chey WD, et al. (2006) Design of treatment trials for functional gastrointestinal disorders. Gastroenterology 130, 1538-1551.

20. Heaton KW, O'Donnell LJ, Braddon FE, et al. (1992) Symptoms of irritable bowel syndrome in a British urban community: consulters and nonconsulters. Gastroenterology 102, $1962-1967$.

21. Guyonnet D, Chassany O, Picard C, et al. (2008) Perceived subject outcomes and impact on health-related quality of life associated with diet using the new Food Benefits Assessment $\left(\mathrm{FBA}^{\odot}\right)$ questionnaire: development and psychometric validation. Public Health Nutr 11, 1163-1172.

22. Dupuy HJ (1984) The psychological general well-being (PGWB) index. In Assessment of Quality of Life in Clinical Trials of Cardiovascular Therapies, chapter 9, pp. 170-184 [NK Wenger, ME Mattson, CF Furberg and J Elinson, editors]. New York: Le Jacq Publishing.

23. Walters SJ \& Brazier JE (2005) Comparison of the minimally important difference for two health state utility measures: EQ-5D and SF-6D. Qual Life Res 14, 1523-1532.

24. Juniper EF, Guyatt GH, Willan A, et al. (1994) Determining a minimal important change in a disease-specific quality of life questionnaire. J Clin Epidemiol 47, 81-87.

25. European Medicines Agency (1998) ICH Topic E 9. Statistical Principles for Clinical Trials. Step 5. Note for guidance on statistical principles for clinical trials (CPMP/ICH/363/96). London: EMEA.

26. Goetze O, Fruehauf H, Pohl D, et al. (2008) Effect of a prebiotic mixture on intestinal comfort and general wellbeing in health. Br J Nut 100, 1077-1085.

27. Hernando-Harder AC, von Bunau R, Nadarajah M, et al. (2008) Influence of E. coli strain Nissle $1917(\mathrm{EcN})$ on intestinal gas dynamics and abdominal sensation. Dig Dis Sci 53, 443-450.

28. McFarland LV \& Dublin S (2008) Meta-analysis of probiotics for the treatment of irritable bowel syndrome. World $J$ Gastroenterol 14, 2650-2661.
29. Cummings JH, Antoine JM, Azpiroz F, et al. (2004) PASSCLAIM - gut health and immunity. Eur J Nutr 43, Suppl. 2, II118-II173.

30. van Kerkhoven LA, Eikendal T, Laheij RJ, et al. (2008) Gastrointestinal symptoms are still common in a general Western population. Neth J Med 66, 18-22.

31. Moayyedi P, Ford AC, Talley NJ, et al. (2008) The efficacy of probiotics in the therapy of irritable bowel syndrome: a systematic review. Gut (epublication ahead of print version 17 December 2008).

32. Nuovo J, Melnikow J \& Chang D (2002) Reporting number needed to treat and absolute risk reduction in randomized controlled trials. JAMA 287, 2813-2814.

33. Altman DG, Schulz KF, Moher D, et al. (2001) The revised CONSORT statement for reporting randomized trials: explanation and elaboration. Ann Intern Med 134, 663-694.

34. Bouglé D, Roland N, Lebeurrier F, et al. (1999) Effect of propionibacteria supplementation on fecal bifidobacteria and segmental colonic transit time in healthy human subjects. Scand J Gastroenterol 34, 144-148.

35. Kim HJ, Camilleri M, McKinzie S, et al. (2003) A randomized controlled trial of a probiotic, VSL\#3, on gut transit and symptoms in diarrhoea-predominant irritable bowel syndrome. Aliment Pharmacol Ther 17, 895-904.

36. Sairanen U, Piirainen L, Grasten S, et al. (2007) The effect of probiotic fermented milk and inulin on the functions and microecology of the intestine. J Dairy Res 74, 367-373.

37. Bartram HP, Scheppach W, Gerlach S, et al. (1994) Does yogurt enriched with Bifidobacterium longum affect colonic microbiology and fecal metabolites in healthy subjects? Am J Clin Nutr 59, 428-432.

38. Collado MC, Moreno Y, Cobo JM, et al. (2006) Molecular detection of Bifidobacterium animalis DN-173010 in human feces during fermented milk administration. Food Res Int 39, 530-535.

39. Parkes GC, Brostoff J, Whelan K, et al. (2008) Gastrointestinal microbiota in irritable bowel syndrome: their role in its pathogenesis and treatment. Am $J$ Gastroenterol 103, $1557-1567$ 